\title{
The period of fibonacci sequences over the finite field of order $p^{2}$
}

\author{
Yasemin Tasyurdu and Inci Gultekin \\ Department of Mathematics, Erzincan University, Erzincan, Turkey \\ Department of Mathematics, Ataturk University, Erzurum, Turkey
}

Received: 11 November 2015, Revised: 10 December 2015, Accepted: 30 December 2015

Published online: 18 February 2016.

\begin{abstract}
In this paper, we obtain the period of Fibonacci sequence in the finite fields of order $p^{2}$ by using equality recursively defined by $F_{n+1}=A_{1} F_{n}+A_{0} F_{n-1}$, for $n>0$, where $F_{0}=0, F_{1}=1$ and $A_{1}, A_{0}$ are generators elements of these fields of order $p^{2}$.
\end{abstract}

Keywords: Fibonacci sequence, period, finite fields.

\section{Introduction}

Generalized Fibonacci sequence have been intensively studied for many years and have become into an interesting topic in Applied Mathematics. Fibonacci sequences and their related higher-order (tribonacci, k-nacci) sequences are generally viewed as sequences of integers. The notation of Wall number was first proposed by D. D. Wall [7] in 1960. In [7], he gave some theorems and properties concerning Wall number of the Fibonacci sequences. K. Lu and J. Wang [5] contributed to the study of the Wall number for the k-step Fibonacci sequences. D. J. De Carli [2] gave a generalized Fibonacci sequences over an arbitrary ring in 1970. Special cases of Fibonacci sequences over an arbitrary ring have been considered by R. G. Bauschman [1], A. F. Horadam [4] and N. N. Vorobyov [6] where this ring was taken to be the set integers. O. Wyler [8] also worked with such a sequence over a particular commutative ring with identity. Classification of finite rings of order $p^{2}$ with $p$ a prime have been studied by B. Fine [3].

A sequence of ring elements is periodic if, after a certain point, it consists only of repetitions of a fixed subsequence. The number of elements in the repeating subsequence is called the period of the sequence. For example, the sequence $a, b, c, d, e, b, c, d, e, b, c, d, e, \ldots$ is periodic after the initial element $a$ and has period 4. A sequence of ring elements is simply periodic with period $k$ if the first $k$ elements in the sequence form a repeating subsequence. For example, the sequence $a, b, c, d, e, f, a, b, c, d, e, f, a, b, c, d, e, f, \ldots$ is simply periodic with period 6.

Definition 1. Let $f_{n}^{k}$ denote the $n$th member of the $k$-step Fibonacci sequence defined as

$$
f_{n}^{(k)}=\sum_{j=1}^{k} f_{n-j}^{(k)} \quad \text { for } \quad n>k
$$


with boundary conditions $f_{i}^{(k)}=0$ for $1 \leq i<k$ and $f_{k}^{(k)}=1$.Reducing this sequence modulo $m$, we can get a repeating sequence, denoted by $f(k, m)=\left(f_{1}^{(k, m)}, f_{2}^{(k, m)}, \ldots, f_{n}^{(k, m)}, \ldots\right)$ where $f_{i}^{(k, m)}=f_{i}^{(k)}(\bmod m)$. Then we have that

$$
f(k, m)=\left(f_{1}^{(k, m)}, f_{2}^{(k, m)}, \ldots, f_{k}^{(k, m)}\right)=(0,0, \ldots, 0,1)
$$

and it has the same recurrence relation as in (1), [5].

Theorem 1. $f(k, m)$ is a periodic sequence [5].

Theorem 2. For any prime $p$, up to isomorphism, the finite 2-generator field of order $p^{2}$ is given by the following presentations [3]:

$$
G F\left(p^{2}\right)=\left\{\begin{array}{c}
\left\langle a, b: p a=p b=0, a^{2}=a, b^{2}=j a, a b=b, b a=b\right\rangle, \\
\text { where } j \text { is not a square in } \mathbb{Z}_{p}, \text { if } p \neq 2 \\
\left\langle a, b: 2 a=2 b=0, a^{2}=a, b^{2}=a+b, a b=b, b a=b\right\rangle, \\
\text { if } p=2
\end{array}\right.
$$

Definition 2. Let $R$ be a ring with identity $I$. The sequence $\left\{M_{n}\right\}$ of elements of $R$ recursively is defined by

$$
M_{n+2}=A_{1} M_{n+1}+A_{0} M_{n} \quad \text { for } \quad n \geq 0
$$

where $M_{o}, M_{1}, A_{0}$ and $A_{1}$ are arbitrary elements of $R$ [2].

Definition 3. A special case of equality (2) is denoted by $\left\{F_{n}\right\}$ and defined by

$$
F_{n+2}=A_{1} F_{n+1}+A_{0} F_{n} \quad \text { for } \quad n \geq 0,
$$

where $F_{o}=0, F_{1}=I$, and $A_{0}, A_{1}$ are arbitrary elements of $R$ [2].

We next denote the identity of the $G F\left(p^{2}\right)$ by 1 .

Theorem 3. If $F_{n+2}=A_{1} F_{n+1}+A_{0} F_{n}$, then $F_{n+2}=F_{n+1} A_{1}+F_{n} A_{0} \quad$ [2].

Theorem 4. Let

$$
G F\left(p^{2}\right)=\left\{\begin{array}{c}
\left\langle a, b: p a=p b=0, a^{2}=a, b^{2}=j a, a b=b, b a=b\right\rangle, \\
\text { where } j \text { is not a square in } \mathbb{Z}_{p}, \text { if } p \neq 2 \\
\left\langle a, b: 2 a=2 b=0, a^{2}=a, b^{2}=a+b, a b=b, b a=b\right\rangle, \\
\text { if } p=2
\end{array}\right.
$$

(i) If $j=p-1$,

$$
0, a, b, 0, b, j a, 0, j a, j b, 0, j b, a, 0, a, b, \ldots
$$

Fibonacci sequences is simple periodic and period is 12 .

(ii) If $j=p-2$,

$$
0, a, b,(j+1) a, 0,(j+1) a,(j+1) b, a, 0, a, b, \ldots
$$

Fibonacci sequences is simple periodic and period is 8 .

(iii) If $j=p-3$,

$$
0, a, b,,(j+1) a,(j+2) b, a, 0, a, b, \ldots
$$

Fibonacci sequences is simple periodic and period is 6 . 
(iv) If $j=p-4$,

$$
\begin{aligned}
& 0, a, b,(j+1) a,(j+2) b, \underbrace{(4 k+1) a,(2 k+1) b,(j-(4 k-1)) a,(j-(2 k-2)) b}_{k=1}, \\
& \underbrace{(4 k+1) a,(2 k+1) b,(j-(4 k-1)) a,(j-(2 k-2)) b}_{k=2}, \ldots, 0,1, b, \ldots
\end{aligned}
$$

Fibonacci sequences is simple periodic and period is $4 p$.

Proof. Let us consider the Definition 1.5. For $F_{n+2}=A_{1} F_{n+1}+A_{0} F_{n}$ where $F_{0}=0, F_{1}=1$ and $A_{1}=b, A_{0}=a, n \geq 0$.

(i) Suppose that a single period of $\bmod (p)$ is partitioned into smaller finite subsequences $A_{0}, A_{1}, A_{2}, \ldots$ as shown below $a=1$ :

$$
\underbrace{0, a, b}_{A_{0}}, \underbrace{0, b, j a}_{A_{1}}, \underbrace{0, j a, j b}_{A_{2}}, \underbrace{0, j b, a}_{A_{3}}, 0, a, b, \ldots
$$

If it is use $j a=b^{2}, j a b=b^{2} b \Longrightarrow j b=b^{3}$

$$
\underbrace{0, a, b}_{A_{0}}, \underbrace{0, b, b^{2}}_{A_{1}}, \underbrace{0, b^{2}, b^{3}}_{A_{2}}, \underbrace{0, b^{3}, a}_{A_{3}}, 0, a, b, \ldots
$$

Each subsequence $A_{i}$ has $\alpha=3$ terms and it contains exactly one zero. Every subsequence $A_{i}$ for $i \geq 1$ is a multiply of $A_{0}$, more precisely, the following congruences hold modulo $p$

$$
\begin{aligned}
& A_{1}=b A_{0} \\
& A_{2}=b^{2} A_{0} \\
& A_{3}=b^{3} A_{0}
\end{aligned}
$$

$$
\begin{aligned}
A_{n-1} & =b^{n-1} A_{0} \\
A_{n} & =b^{n} A_{0}
\end{aligned}
$$

Now, the last term in $A_{n-1}$ is $b^{n}$, the last term in $A_{0}$ is $b$ and the last term in $A_{3}$ is $b^{4}=a=1$, i.e order of $b$ is 4 . If the number of subsequences $A_{i}$ is $\beta=4$, clearly it follows that Fibonacci sequence is simple periodic and period is $\alpha . \beta=3.4=12$.

(ii) Suppose that a single period of $\bmod (p)$ is partitioned into smaller finite subsequences $A_{0}, A_{1}, A_{2}, \ldots$ as shown below $a=1$ :

$$
0, a, b,(j+1) a, 0,(j+1) a,(j+1) b, a, 0, a, b, \ldots
$$

If it is use $j a=b^{2}, j a b=b^{2} b \Longrightarrow j b=b^{3}, j b^{2}=4 a=b^{4}, 4 a b=4 b=b^{5}, \ldots,(j+1) a=b^{p-1},(j+1) b=b^{p}, \ldots$ Then,

$$
\underbrace{0, a, b, b^{p-1}}_{A_{0}}, \underbrace{0, b^{p-1}, b^{p}, b^{2 p-2}=a}_{A_{1}}, 0, a, b, \ldots
$$


Each subsequence $A_{i}$ has $\alpha=4$ term and it contains exactly one zero. Every subsequence $A_{1}$ is a multiply of $A_{0}$, more precisely, the following congruences hold modulo $p$

$$
A_{1} \equiv b^{p-1} A_{0}
$$

Now, the last term in $A_{o}$ is $b^{p-1}$ and the last term in $A_{1}$ is $b^{2 p-2}=a=1$, i.e., order of $b$ is $2 p-2$. If number of subsequences $A_{i}$ is $\beta=2$. Clearly, it follows that period is $\alpha$. $\beta$. So, Fibonacci sequence is simple periodic and period is $4.2=8$.

(iii) If $j=p-3$,

$$
\underbrace{0, a, b,(j+1) a,(j+2) b, a}_{A_{0}}, 0, a, b, \ldots
$$

It is clear that only subsequence $A_{0}$ has $\alpha=6$ term and it contains exactly one zero. Thus, Fibonacci sequence is simple periodic and period is $1.6=6$.

(iv) Suppose that a single period of $\bmod (p)$ is partitioned into smaller finite subsequences $A_{0}, A_{1}, A_{2}, \ldots$ as shown below $a=1$ :

$$
\begin{aligned}
& 0, a, b,(j+1) a,(j+2) b, \underbrace{(4 k+1) a,(2 k+1) b,(j-(4 k-1)) a,(j-(2 k-2)) b}_{k=1}, \\
& \underbrace{(4 k+1) a,(2 k+1) b,(j-(4 k-1)) a,(j-(2 k-2)) b}_{k=2}, \ldots, 0, a, b, \ldots
\end{aligned}
$$

and

$$
\begin{aligned}
& \underbrace{0, a, b,(j+1) a,(j+2) b, \underbrace{(4 k+1) a,(2 k+1) b,(j-(4 k-1)) a,(j-(2 k-2)) b}_{k=1}}_{A_{0}}, \\
& \underbrace{\underbrace{4 k+1) a,(2 k+1) b,(j-(4 k-1)) a,(j-(2 k-2)) b}_{k=2}, \ldots, \underbrace{(4 k+1) a,(2 k+1) b}_{k=r}}_{A_{0}}, \\
& \underbrace{\underbrace{(j-(4 k-1)) a,(j-(2 k-2)) b}_{A_{1}}, \underbrace{(4 k+1) a,(2 k+1) b}_{k=r+1}(4 k+1) a,(2 k+1) b, \ldots, \underbrace{(4 k+1) a,(2 k+1) b}_{k=s}}_{k=r}, \\
& \underbrace{\underbrace{(j-(4 k-1)) a,(j-(2 k-2)) b}_{A_{2}}, \underbrace{(4 k+1) a,(2 k+1) b}_{k=s+1}(4 k+1) a,(2 k+1) b, \ldots, \underbrace{(4 k+1) a,(2 k+1) b}_{k=t}}_{k=s}, \\
& \underbrace{\underbrace{(j-(4 k-1)) a,(j-(2 k-2)) b}_{k=t}, \underbrace{(4 k+1) a,(2 k+1) b}_{k=t+1}(4 k+1) a,(2 k+1) b, \ldots, \underbrace{(4 k+1) a,(2 k+1) b}_{k=u}}_{A_{3}},
\end{aligned}
$$

Each subsequences $A_{i}$ has $p$ term and it contains exactly one zero. If $j=4 k-1, F_{p n}=0, F_{p n+1}=(j-(2 k-2)) b, 1 \leq$ $n \leq 3$, then

$$
F_{4 p}=0, F_{4 p+1}=a, F_{4 p+2}=b, \ldots
$$

Thus, Fibonacci sequence is simple periodic and period is $4 p$. 
Example 1. (i) For $p=11$, the presentation of $G F\left(11^{2}\right)$

$$
G F\left(11^{2}\right)=\left\langle a, b: 11 a=11 b=0, a^{2}=a, b^{2}=j a, a b=b, b a=b\right\rangle
$$

$j$ is not square an element in the $\mathbb{Z}_{11}$. If $p=11$, the non-square elements of $\mathbb{Z}_{11}$ can be calculated as follows.

$$
1^{2}=1,2^{2}=4,3^{2}=9,4^{2}=5,5^{2}=3,6^{2}=3,7^{2}=5,8^{2}=9,9^{2}=4
$$

where $\mathbb{Z}_{11}=\{\overline{0}, \overline{1}, \overline{2}, \overline{3}, \overline{4}, \overline{5}, \overline{6}, \overline{7}, \overline{8}, \overline{9}, \overline{10}\}$. After this point, the numbers repeat. For example $10^{2}=(-1)^{2}=1^{2}=1$.

Therefore the set of elements that are not square in the $\mathbb{Z}_{11}$ is $\{\overline{2}, \overline{6}, \overline{7}, \overline{8}, \overline{10}\}$ and the set of elements that are square in the $\mathbb{Z}_{11}$ is $\{\overline{0}, \overline{1}, \overline{3}, \overline{4}, \overline{5}, \overline{9}\}$. From Theorem 1.7. i., $j=11-1=10$

$$
G F\left(11^{2}\right)=\left\langle a, b: 11 a=11 b=0, a^{2}=a, b^{2}=10 a, a b=b, b a=b\right\rangle
$$

Let us consider the Definition 1.5. For $F_{n+2}=A_{1} F_{n+1}+A_{0} F_{n}$ where $F_{0}=0, F_{1}=1$ and $A_{1}=b, A_{0}=a, n \geq 0$.

$$
\begin{aligned}
& 0,1,=a, b, b^{2}+a^{2}=10 a+a=11 a=0, b a=b, b^{2}=10 a, \\
& 10 a b+b a=11 b=0,10 a^{2}=10 a, 10 a b=10 b, 10 b^{2}+10 a^{2}=110 a=0, \\
& 10 b a=10 b, 10 b^{2}=100 a=a, a b+10 b a=11 b=0, a^{2}=a, a b=b, \ldots
\end{aligned}
$$

From relations in the $G F\left(11^{2}\right)$, we have follows

$$
b^{2}=10 a, b^{3}=10 a b=10 b, b^{4}=10 b^{2}=100 a=a
$$

Then the sequence is

$$
\underbrace{0, a, b}_{A_{0}}, \underbrace{0, b, b^{2}}_{A_{1}}, \underbrace{0, b^{2}, b^{3}}_{A_{2}}, \underbrace{0, b^{3}, a}_{A_{3}}, 0, a, b, \ldots
$$

Each subsequence has 3 terms and the number of subsequences 4 . Thus, Fibonacci sequence is simple periodic and period is $3.4=12$.

(ii) For $p=13$, the presentation of $G F\left(13^{2}\right)$

$$
G F\left(13^{2}\right)=\left\langle a, b: 13 a=13 b=0, a^{2}=a, b^{2}=j a, a b=b, b a=b\right\rangle
$$

$j$ is not square an element in the $\mathbb{Z}_{13}$. Set of elements that are not square in the $\mathbb{Z}_{13}$ is $\{\overline{2}, \overline{5}, \overline{6}, \overline{7}, \overline{8}, \overline{11}\}$ and the set of elements that are square in the $\mathbb{Z}_{13}$ is $\{\overline{0}, \overline{1}, \overline{3}, \overline{4}, \overline{9}, \overline{10}, \overline{12}\}$. From Theorem 1.7. (ii), $j=13-2=11$

$$
G F\left(13^{2}\right)=\left\langle a, b: 13 a=13 b=0, a^{2}=a, b^{2}=11 a, a b=b, b a=b\right\rangle
$$


Let us consider the Definition 1.5. For $F_{n+2}=A_{1} F_{n+1}+A_{0} F_{n}$ where $F_{0}=0, F_{1}=1$ and $A_{1}=b, A_{0}=a, n \geq 0$.

$$
\begin{aligned}
& 0,1, a, b, b^{2}+a^{2}=11 a+a=12 a, 12 a b+b a=13 b=0,12 a^{2}=12 a, \\
& 12 a b=12 b, 12 b^{2}+12 a^{2}=132 a+12 a=144 a=a, a b+12 b a=0, \\
& a^{2}=a, a b=b, \ldots
\end{aligned}
$$

From relations in the $G F\left(13^{2}\right)$, we have follows

$$
\begin{aligned}
& b^{2}=11 a, b^{3}=11 a b=11 b, b^{4}=11 b^{2}=121 a=4 a, b^{5}=4 a b=4 b, \\
& b^{6}=5 a, b^{7}=5 b, b^{8}=3 a, b^{9}=3 b, b^{10}=7 a, b^{11}=7 b, \\
& b^{17}=9 b, b^{18}=99 a=8 a, b^{19}=8 b, b^{20}=88 a=10 a, b^{21}=10 b, \\
& b^{22}=110 a=6 a, b^{23}=6 a b=6 b, b^{24}=66 a=a, \ldots
\end{aligned}
$$

That is $b^{2 p-2}=b^{26-2}=b^{24}=a$. Then the sequence is

$$
\begin{aligned}
& \underbrace{0, a, b, 12 a}_{A_{0}}, \underbrace{0,12 a, 12 b, a}_{A_{1}}, 0, a, b, \ldots \\
& \underbrace{0, a, b, b^{12}}_{A_{0}}, \underbrace{0, b^{12}, b^{13}, b^{24}}_{A_{1}}, 0, a, b \ldots
\end{aligned}
$$

Each subsequence has 4 terms and the number of subsequences 2 . Thus, Fibonacci sequence is simple periodic and period is $2.4=8$.

(iii) For $p=17$, the presentation of $G F\left(17^{2}\right)$

$$
G F\left(17^{2}\right)=\left\langle a, b: 17 a=17 b=0, a^{2}=a, b^{2}=j a, a b=b, b a=b\right\rangle
$$

$j$ is not square an element in the $\mathbb{Z}_{17}$. Set of elements that are not square in the $\mathbb{Z}_{17}$ is $\{\overline{3}, \overline{5}, \overline{6}, \overline{7}, \overline{10}, \overline{11}, \overline{12}, \overline{14}\}$ and the set of elements that are square in the $\mathbb{Z}_{17}$ is $\{\overline{0}, \overline{1}, \overline{2}, \overline{4}, \overline{8}, \overline{9}, \overline{13}, \overline{15}, \overline{16}\}$. From Theorem 1.7. iii., $j=17-3=14$

$$
G F\left(17^{2}\right)=\left\langle a, b: 17 a=17 b=0, a^{2}=a, b^{2}=14 a, a b=b, b a=b\right\rangle
$$

Let us consider the Definition 1.5. For $F_{n+2}=A_{1} F_{n+1}+A_{0} F_{n}$ where $F_{0}=0, F_{1}=1$ and $A_{1}=b, A_{0}=a, n \geq 0$.

$$
\begin{aligned}
& 0,1,=a, b, b^{2}+a^{2}=14 a+a=15 a, 15 b a+a b=16 b, \\
& 16 b^{2}+15 a^{2}=224 a+15 a=239 a=a, b a+16 a b=b+16 b=17 b=0, \\
& b 0+a a=a^{2}=a, b a+a 0=b, b b+a a=b^{2}+a^{2}=14 a+a=15 a, \ldots
\end{aligned}
$$

Then the sequence is

$$
\underbrace{0, a, b, 15 a, 16 b, a}_{A_{0}}, 0, a, b, 15 a, \ldots
$$

Subsequence has 6 terms and there is a subsequence. Thus, Fibonacci sequence is simple periodic and period is 6 . 
(iv) For $p=19$, the presentation of $G F\left(19^{2}\right)$

$$
G F\left(19^{2}\right)=\left\langle a, b: 19 a=19 b=0, a^{2}=a, b^{2}=j a, a b=b, b a=b\right\rangle
$$

$j$ is not square an element in the $\mathbb{Z}_{19}$. Set of elements that are not square in the $\mathbb{Z}_{19}$ is $\{\overline{2}, \overline{3}, \overline{7}, \overline{8}, \overline{10}, \overline{12}, \overline{13}, \overline{14}, \overline{15}, \overline{18}\}$ and the set of elements that are square in the $\mathbb{Z}_{19}$ is $\{\overline{0}, \overline{1}, \overline{4}, \overline{5}, \overline{6}, \overline{9}, \overline{11}, \overline{16}, \overline{17}\}$. From Theorem 1.7. (iii), $j=19-4=$ 15

$$
G F\left(19^{2}\right)=\left\langle a, b: 19 a=19 b=0, a^{2}=a, b^{2}=15 a, a b=b, b a=b\right\rangle
$$

Let us consider the Definition 1.5. For $F_{n+2}=A_{1} F_{n+1}+A_{0} F_{n}$ where $F_{0}=0, F_{1}=1$ and $A_{1}=b, A_{0}=a, n \geq 0$. It is use relations to $G F\left(19^{2}\right)$,

$\underbrace{0, a, b, 16 a, 17 b, 5 a, 3 b, 12 a, 15 b, 9 a, 5 b, 8 a, 13 b, 13 a, 7 b, 4 a, 11 b, 17 a, 9 b}_{A_{0}}$

$\underbrace{0,9 b, 2 a, 11 b, 15 a, 7 b, 6 a, 13 b, 11 a, 5 b, 10 a, 15 b, 7 a, 3 b, 14 a, 17 b, 3 a, b, 18 a}_{A_{1}}$

$\underbrace{0,18 a, 18 b, 3 a, 2 b, 14 a, 16 b, 7 a, 4 b, 10 a, 14 b, 11 a, 6 b, 6 a, 12 b, 15 a, 8 b, 2 a, 10 b}$

$A_{2}$

$\underbrace{0,10 b, 17 a, 8 b, 4 a, 12 b, 13 a, 6 b, 8 a, 14 b, 9 a, 4 b, 12 a, 16 b, 5 a, 2 b, 16 a, 18 b, a}$

$A_{3}$

$0, a, \ldots$

For $k=4, \quad j=4.4-1, F_{19.1}=0, \quad F_{19.1+1}=F_{20}=(15-(2.4-2)) b=9 b, \quad 1 \leq n \leq 3, \quad F_{4.19}=0, \quad F_{4.19+1}=$ $a, F_{4.19+2}=b$.

It is clear that subsequence $A_{i}, 0 \leq i \leq 3$, has $p=19$ term and it contains exactly one zero. Thus, Fibonacci sequence is simple periodic and period is $4 p=4.19=76$.

\section{Conclusion}

For any prime $p$, up to isomorphism, it can be seen that the period of the Fibonacci sequence $G F\left(p^{2}\right)$ of field of order $p^{2}$ is determined by $j$ in the presentation of $G F\left(p^{2}\right)$. Consider $p=11$ :

(i) From Example i., we have that the period of the Fibonacci sequence is 12 for $j=p-1, p=11$.

(ii) We not use Theorem 1.7., ii. for $p=11$ because $j=p-2=11-2=9 \notin\{\overline{2}, \overline{6}, \overline{7}, \overline{8}, \overline{10}\}$ for Theorem 1.7., ii. where $j$ is not square an element in the $\mathbb{Z}_{11}, j \in\{\overline{2}, \overline{6}, \overline{7}, \overline{8}, \overline{10}\}$.

(iii) Let us consider the Theorem 1.7., iii. for $j=p-3=11-3=8 \in\{\overline{2}, \overline{6}, \overline{7}, \overline{8}, \overline{10}\}$

$$
G F\left(11^{2}\right)=\left\langle a b: 11 a=11 b=0, a^{2}=a, b^{2}=8 a, a b=b, b a=b\right\rangle
$$

From Definition 1.5. For $F_{n+2}=A_{1} F_{n+1}+A_{0} F_{n}$ where $F_{0}=0, F_{1}=1$ and $A_{1}=b, A_{0}=a, n \geq 0$. 


$$
\begin{aligned}
& 0,1=a, b, b^{2}+a^{2}=8 a+a=9 a, 9 b a+a b=10 b \\
& 10 b^{2}+9 a^{2}=80 a+9 a=a, b a+10 a b=b+10 b=11 b=0 \\
& b 0+a a=a^{2}=a, b a+a 0=b, b b+a a=b^{2}+a^{2}=8 a+a=9 a, \ldots
\end{aligned}
$$

Then the sequence is

$$
\underbrace{0, a, b, 9 a, 10 b, a}_{A_{0}}, 0, a, b, 9 a, \ldots
$$

Subsequence has 6 terms and there is a subsequence. Thus, Fibonacci sequence is simple periodic and period is 6 .

(iv) If it is use Theorem 1.7., iv. for $j=p-4=11-4=7$, it can be seen clearly that the period of the Fibonacci sequence is 44 .

Consequently, the period of the Fibonacci sequence is 12 for $j=p-1$, the period of the Fibonacci sequence is 6 for $j=p-3$ and the period of the Fibonacci sequence is 44 for $, j=p-4, p=11$.

\section{Acknowledgment}

The research of Y. Tasyurdu was supported by Erzincan University Rectorship under "The Scientific and Research Project of Erzincan University", project no. FEN-A-240215-0122.

\section{References}

[1] R. G. Buschaman, “Fibonacci Numbers, Chebyschev Polynomials, Generalizations and Difference Equations” Fibonacci Qartely, Vol.1, No.4, (1963), pp 1-7.

[2] D. J. Decarl1, "A Generalized Fibonacci Sequence Over An Arbitrary Ring” The Fibonacci Quartely, Vol.8, No.2 (1970), pp 182184.

[3] B. Fine, “Classification of Finite Rings Of order” Mathematical Association of America, Vol.66, No. 4, (1993), pp 248-252.

[4] A. F. Horadam, “ A Generalized Fibonacci Sequence” American Mathmatical Montly, 68 (1961), pp 445-459.

[5] K. Lü, J. Wang. “ k-step Fibonacci squence modulo m” Util. Math. 71(2007), pp 169-178.

[6] N. N. Vorobyov, The Fibonacci Numbers, translated from the Russian by Normal D. Whaland, Jr., and Olga A. Tittlebaum, D.C.Heath and Co., Boston,(1963).

[7] D. D. Wall, “ Fibonacci series modulo m”, American Mathematical Montly, 67 (1960), pp 525-532

[8] O. Wyler, "On Second Order Recurrences” American Mathmatical Montly,72(1965), pp 500-506. Author, Title, Journal/Editor, (year). 